\title{
The relationship of the musculocutaneous nerve to the brachial plexus evaluated by MRI
}

\author{
Trygve Kjelstrup $^{1,2} \cdot$ Axel R. Sauter ${ }^{3}$ Per K. Hol ${ }^{2,4}$
}

Received: 23 May 2015/Accepted: 15 November 2015/Published online: 19 November 2015

(c) The Author(s) 2015. This article is published with open access at Springerlink.com

\begin{abstract}
Axillary plexus blocks (AXB) are widely used for upper limb operations. It is recommend that AXB should be performed using a multiple injection technique. Information about the course and position of the musculocutaneous nerve (MCN) is of relevance for AXB performance. The objective of this study was to examine the position of the MCN and its relationship to the axillary sheath using MRI. 54 patients underwent an AXB with $40 \mathrm{ml}$ of local anaesthetic before MRI examination. The course of the MCN and the position where it left the axillary sheath and perforated the coracobrachial muscle (MCN exit point), in relation to the axillary artery and the block needle insertion point in the axillary fold, were recorded. The MCN was seen clearly in 23 , partly in 26 , and not identified in five patients at the MCN exit point. The mean distance from the insertion point of the block needle in the axillary fold to the MCN exit point was $36.8 \mathrm{~mm}(\mathrm{SD}=18.9$, range: $0-90.5)$. In 37 patients the MCN exit point was positioned inside the $\mathrm{Q}_{1}$ quadrant (lateral anterior to the axillary artery) and in 11 patients inside the $\mathrm{Q}_{2}$ quadrant (medial anterior to the axillary artery). There is a wide variability as to where the
\end{abstract}

Trygve Kjelstrup

trygve.kjelstrup@labmed.uio.no

1 Department of Anaesthesiology, Diakonhjemmet Hospital, $\mathrm{Pb} 23$ Vinderen, 0319 Oslo, Norway

2 The Intervention Centre, Oslo University Hospital, Rikshospitalet, Oslo, Norway

3 Division of Emergencies and Critical Care, Department of Anaesthesiology, Oslo University Hospital, Rikshospitalet, Oslo, Norway

4 Institute of Clinical Medicine, University of Oslo, Oslo, Norway musculocutaneous nerve (MCN) leaves the axillary sheath. Therefore multiple injection techniques, or the use of a proximally directed catheter, should be appropriate to block the MCN.

Keywords Brachial plexus - Musculocutaneous nerve · MRI scan

\section{Introduction}

Axillary plexus blocks (AXB) are widely used as an anaesthetic method for upper limb operations. It is recommend that AXB should be performed using a multiple injection technique [1]. At terminal nerve level, the musculocutaneous nerve (MCN) is usually positioned outside the axillary sheath. There is an ongoing discussion as to whether a selective block of the MCN is necessary to achieve a successful AXB [2,3]. The objective in this study was to examine the position of the MCN and its relationship to the axillary sheath using MRI. Information about the course of the MCN can indicate how, and where, the MCN can be blocked when performing AXB.

\section{Methods}

Ethical approval for this study (Ethical Committee No S-04115) was provided by the Committee for Medical Research Ethics, Region South East (REK Sør-Øst), Pb 1130 Blindern, 0318 Oslo, Norway on June 8th, 2004 as for a previous study [4].

The study was conducted at Oslo University Hospital, Rikshospitalet, in the period November 10th, 2014-February 1st, 2015. Forty five patients from a study on 
axillary brachial plexus blocks [5] and nine patients from a pilot study [4] were analysed as one group using the existing MRI data.

All patients underwent an AXB with $40 \mathrm{ml}$ of local anaesthetic (LA) injected before MRI scanning (Achieva 3T, Philips Healthcare, The Netherlands) was performed $[4,5]$. MRI scanning was performed immediately after completion of the block-procedures.

The patients were examined in the horizontal supine position with the arms adducted. T1- and T2-weighted images were obtained according with a previously described protocol [4], and the images underwent a consensus assessment by the three authors.

The course of the MCN and the surrounding anatomy was analysed in the short axis view (cross-sectional, Fig. 1) and in the long axis view (coronal, Fig. 2). The MCN exit point was defined as the position where the nerve left the neurovascular bundle into the coracobrachial muscle (Fig. 1).

The MCN exit point was measured with reference to the top of the humeral head (Fig. 2). Similarly, the most cranial part of the axillary fold was defined as the block needle insertion point, and the distance from this point to the top of the humeral head was recorded (Fig. 2). The difference between these two measurements gave the distance from the block needle insertion point to the MCN exit point.

The visibility assessment was performed at the MCN exit point and was scored as $0=$ not visible, $1=$ partly visible and $2=$ clear visible.

The positions of the MCN exit points were plotted graphically. The estimated diameters were $8 \mathrm{~mm}$ for the artery and $3 \mathrm{~mm}$ for the nerves (Figs. 3 and 4).

The MCN exit point was also described by the four quadrant positions: $\mathrm{Q}_{1}=$ the lateral anterior, $\mathrm{Q}_{2}=$ the medial anterior, $\mathrm{Q}_{3}=$ the medial posterior and $\mathrm{Q}_{4}=$ the lateral posterior quadrant (Fig. 4). The angel and distance from the mid axis of the axillary artery to the MCN exit point was recorded, and a lateral horizontal line towards the humerus was defined as the zero angel line (Fig. 1).

\subsection{Statistics}

For statistical analysis, we used SPSS version 22 (IBM Corporation, Armonk, New York, USA). Data were described by mean, standard deviation (SD), range and counts.

\section{Results}

Of the 54 patients, 30 had a plexus block on the right and 24 on the left side. In 23 patients the MCN exit point was clearly and 26 patients partly visible. In 5 patients the MCN exit point could not be identified.

The mean distance from the top of the humeral head to the insertion point of the block needle was $98.4 \mathrm{~mm}$ ( $\mathrm{SD}=10.9$, range: $81.4-132$ ). The $\mathrm{MCN}$ exit point was positioned at a mean distance of $61.5 \mathrm{~mm}(\mathrm{SD}=17.6$, range: 29.6-106.7) from the humeral head. Thus, the mean distance from the insertion point of the block needle in the axillary fold to the MCN exit point was $36.8 \mathrm{~mm}$ ( $\mathrm{SD}=18.9$, range: 0-90.5; Fig. 3).

The mean angel from the lateral horizontal line to the $\mathrm{MCN}$ exit point was $69.4^{\circ}\left(\mathrm{SD}=39.7^{\circ}\right.$, range: $-8.1^{\circ}$ to $160.2^{\circ}$ ) for all patients (Fig. 4). In 37 patients the MCN exit point was positioned inside the $\mathrm{Q}_{1}$ quadrant (lateral anterior to the axillary artery) and in 11 patients inside the $\mathrm{Q}_{2}$ quadrant (medial anterior to the axillary artery). In only one patient the $\mathrm{MCN}$ exit point was found in the $\mathrm{Q}_{4}$ quadrant at $-8.1^{\circ}$ to the zero angel line (Fig. 4). The
Fig. 1 MRI of the right axilla, cross-sectional view. T2weighted image with fat suppression from a patient with a successful sensory block after a single local anaesthetic (LA) injection. The LA appears white. The musculocutaneous nerve $(\mathrm{MCN})$ is clearly seen before entering the coracobrachial muscle. The lateral horizontal line $(\mathrm{red})$ towards the humerus head was defined as the zero angel line

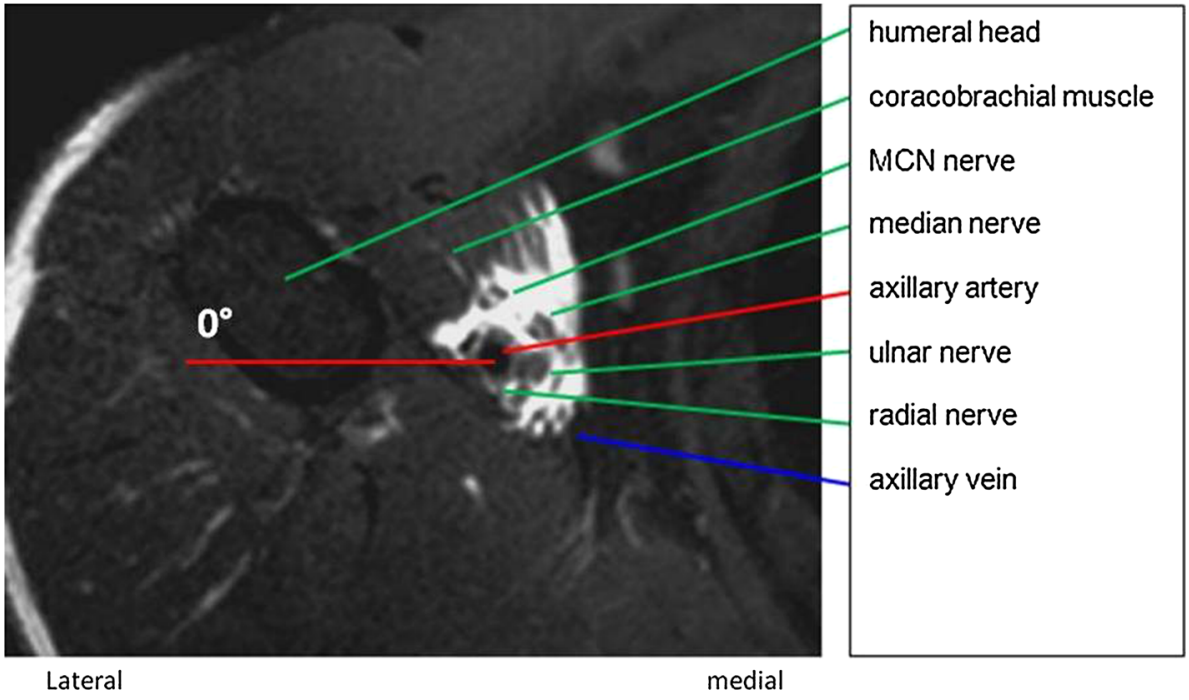

medial 
Fig. 2 MRI of right shoulder, coronal view, in one of the patients. T1-weighted image. With a reference point at the top of the humeral head, the distances (blue line, green line) to the MCN exit point $(N)$ and the insertion point of the block needle $(I)$ were measured. The humeral shaft (red line) served as reference line

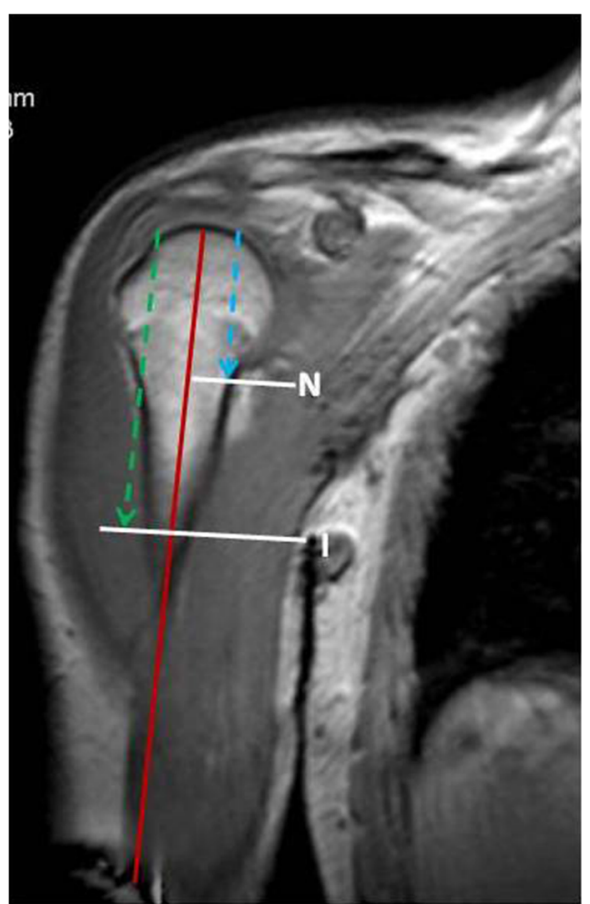

$\mathrm{N}$ : Exit point of the musculocutaneous nerve into the coracobrachial muscle (MCN exit point)

I : Block needle insertion point

Blue line : Distance to $\mathrm{MCN}$ exit point

Green line : Distance to I

Red line : Midaxis of humerus

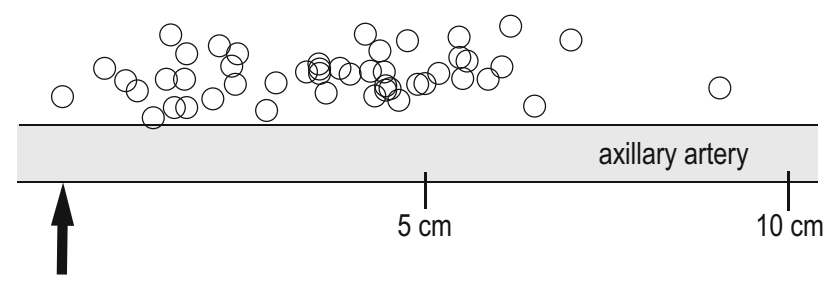

Fig. 3 The exit points of the musculocutaneous nerve into the coracobrachial muscle in relation to the insertion point of the block needle (arrow) in the axillary fold, and in relation to the centre of the artery

average distance from the centre of the artery to the $\mathrm{MCN}$ exit point was $10.8 \mathrm{~mm}(\mathrm{SD}=3.0$, range: $4.9-17.5)$.

\section{Discussion}

The MRI of the axillary brachial plexus from 54 patients showed a wide variability as to where the musculocutaneous nerve $(\mathrm{MCN})$ leaves the axillary sheath. The $\mathrm{MCN}$ exit points were mainly localized in the lateral anterior $\left(\mathrm{Q}_{1}\right)$ and the medial anterior $\left(\mathrm{Q}_{2}\right)$ quadrant and spread over a distance of approximately $10 \mathrm{~cm}$ proximally to the axillary fold.

The large distance between the MCN exit point and the insertion point of the block needle in the axillary fold, which we found in our study, can explain the incomplete success rates in some axillary blocks techniques when the $\mathrm{MCN}$ is not selectively blocked. Block success rates of the

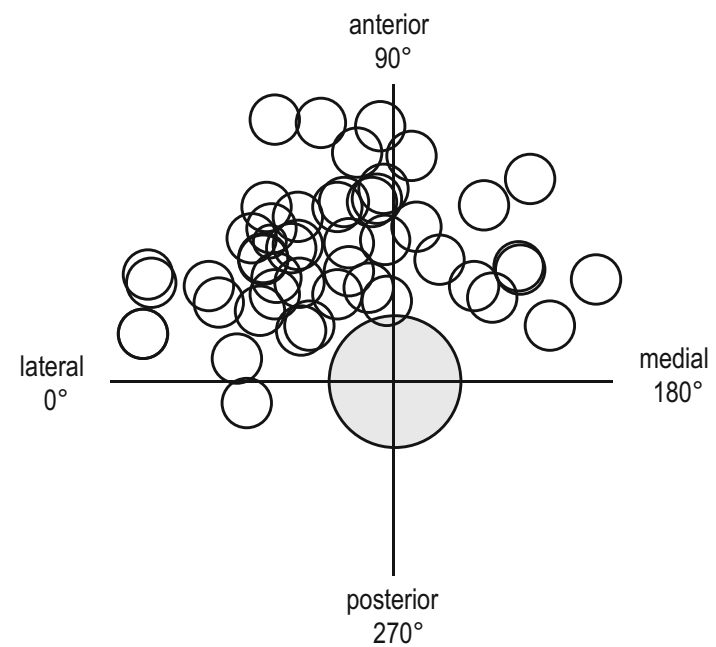

Fig. 4 The exit points of the musculocutaneous nerve into the coracobrachial muscle in relation to the quadrants and the axillary artery (centre). The four quadrants: $\mathrm{Q}_{1}=$ the lateral anterior $\left(0^{\circ}\right.$ $\left.90^{\circ}\right), \mathrm{Q}_{2}=$ the medial anterior $\left(90^{\circ}-180^{\circ}\right), \mathrm{Q}_{3}=$ the medial posterior $\left(180^{\circ}-270^{\circ}\right)$ and $\mathrm{Q}_{4}=$ the lateral posterior $\left(270^{\circ}-360^{\circ}\right)$ quadrant

MCN can range from 27 to $40 \%$ as seen in a study without a selective block of the nerve [6]. With a selective block on the other hand, success rates for MCN blocks above $90 \%$ are achieved [6-8].

According to the concept of a continuous neurovascular sheath, a sufficiently high injection volume should give an adequate proximal spread to the MCN [9]. However, several studies have demonstrated that the brachial plexus 
sheath is divided by connective tissue septa into multiple compartments $[10,11]$. LA spread, especially in the crosssectional plane, can be inhibited. The wide spread of the MCN exit points in the two anterior quadrants favour a multiple injection technique, or a selective block, in order to achieve a successful AXB.

In our previous study, block success of the MCN was $100 \%$ when an LA volume of $40 \mathrm{ml}$ was applied with multiple injections through a proximal catheter combined with two deposits lateral and medial to the axillary artery (without blocking the MCN selectively). When only a proximal catheter was used for LA injection, the success rate for MCN block decreased to $73 \%$ [5].

Non-ultrasound-guided and single injection axillary plexus block techniques have typically used high doses of LA [12-14]. We therefore considered the $40 \mathrm{ml}$ LA volume used in our study as adequate in order to fill up the axillary sheath [15]. When nerves are detected individually by ultrasound guidance or electrical nerve stimulations, lower LA volumes are appropriate [12, 16, 17].

Our study has several limitations. MRI measurements are substantially observer dependant. Therefore the images were discussed among the three authors, and a consensus agreement was achieved. In five of the 54 patients $(9 \%)$ the MCN exit point could not be identified. One reason for our missing data may be that the MCN exit point was outside the scanning field. However, the MCN can sometimes be missing, or it demonstrates unusual anatomy with an alternative course, passing over the surface of the coracobrachial muscle instead of perforating it [18-20]. In the study of Guerri-Guttenberg et al. [19] the MCN was absent at a frequency of $3.6 \%$, and in $11 \%$ of the dissections the nerve did not perforate the coracobrachial muscle.

The MRI examination was performed just after the LA had been injected. The distortion of the anatomy [4], due to the injection, may have altered the MRI measurements. Especially the distance between the MCN exit point and the artery could be increased.

Because of the closed scanner construction, our MRI examinations were performed with the arm adducted. Distances and nerve positions might differ when the arm is abducted, as in the typical position when performing the AXB.

\section{Conclusion}

There is a wide variability as to where the musculocutaneous nerve $(\mathrm{MCN})$ leaves the axillary sheath. Therefore multiple injection techniques, or the use of a proximally directed catheter, should be appropriate to block the MCN.
Acknowledgments Assistance with this study: We thank Mike Dodgson, BSc MB ChB FRCA for review of the language.

Funding This study did not receive any funding.

\section{Compliance with ethical standards}

Conflict of interest The authors declare that they have no conflict of interest.

Ethical approval All procedures performed in studies involving human participants were in accordance with the ethical standards of the institutional and or national research committee and with the 1964 Helsinki declaration and its later amendments or comparable ethical standards.

Open Access This article is distributed under the terms of the Creative Commons Attribution 4.0 International License (http://crea tivecommons.org/licenses/by/4.0/), which permits unrestricted use, distribution, and reproduction in any medium, provided you give appropriate credit to the original author(s) and the source, provide a link to the Creative Commons license, and indicate if changes were made.

\section{References}

1. Chin KJ, Alakkad H, Cubillos JE. Single, double or multipleinjection techniques for non-ultrasound guided axillary brachial plexus block in adults undergoing surgery of the lower arm. Cochrane Database Syst Rev. 2013;8:CD003842.

2. Bernucci F, Gonzalez AP, Finlayson RJ, de Tran QH. A prospective, randomized comparison between perivascular and perineural ultrasound-guided axillary brachial plexus block. Reg Anesth Pain Med. 2012;37:473-7.

3. de Tran QH, Pham K, Dugani S, Finlayson RJ. A prospective, randomized comparison between double-, triple-, and quadrupleinjection ultrasound-guided axillary brachial plexus block. Reg Anesth Pain Med. 2012;37:248-53.

4. Kjelstrup T, Courivaud F, Klaastad O, et al. High-resolution MRI demonstrates detailed anatomy of the axillary brachial plexus. A pilot study. Acta Anaesthesiol Scand. 2012;56:914-9.

5. Kjelstrup T, Hol PK, Courivaud F, et al. MRI of axillary brachial plexus blocks: a randomised controlled study. Eur J Anaesthesiol. 2014;11:611-9.

6. Rodriguez J, Taboada M, Del RS, et al. A comparison of four stimulation patterns in axillary block. Reg Anesth Pain Med. 2005;30:324-8.

7. de Tran QH, Clemente A, Tran DQ, Finlayson RJ. A comparison between ultrasound-guided infraclavicular block using the "double bubble" sign and neurostimulation-guided axillary block. Anesth Analg. 2008;107:1075-8.

8. Rodriguez J, Taboada M, Oliveira J, et al. Radial plus musculocutaneous nerve stimulation for axillary block is inferior to triple nerve stimulation with $2 \%$ mepivacaine. J Clin Anesth. 2008;20:253-6.

9. Winnie AP, Radonjic R, Akkineni SR, Durrani Z. Factors influencing distribution of local anesthetic injected into the brachial plexus sheath. Anesth Analg. 1979;58:225-34.

10. Klaastad O, Smedby O, Thompson GE, et al. Distribution of local anesthetic in axillary brachial plexus block: a clinical and magnetic resonance imaging study. Anesthesiology. 2002;96:1315-24.

11. Thompson GE, Rorie DK. Functional anatomy of the brachial plexus sheaths. Anesthesiology. 1983;59:117-22. 
12. Koscielniak-Nielsen ZJ, Rotboll NP, Sorensen T, Stenor M. Low dose axillary block by targeted injections of the terminal nerves. Can J Anaesth. 1999;46:658-64.

13. Sia S, Bartoli M, Lepri A, et al. Multiple-injection axillary brachial plexus block: a comparison of two methods of nerve localization-nerve stimulation versus paresthesia. Anesth Analg. 2000;91:647-51.

14. Sia S, Lepri A, Ponzecchi P. Axillary brachial plexus block using peripheral nerve stimulator: a comparison between double- and triple-injection techniques. Reg Anesth Pain Med. 2001;26: 499-503.

15. De Jong R. Axillary block of the brachial plexus. Anesthesiology. 1961;22:215-25.

16. Casati A, Danelli G, Baciarello M, et al. A prospective, randomized comparison between ultrasound and nerve stimulation guidance for multiple injection axillary brachial plexus block. Anesthesiology. 2007;106:992-6.

17. Koscielniak-Nielsen ZJ. Multiple injections in axillary block: where and how many? Reg Anesth Pain Med. 2006;31:192-5.

18. Flatow EL, Bigliani LU, April EW. An anatomic study of the musculocutaneous nerve and its relationship to the coracoid process. Clin Orthop Relat Res. 1989;244:166-71.

19. Guerri-Guttenberg RA, Ingolotti M. Classifying musculocutaneous nerve variations. Clin Anat. 2009;22:671-83.

20. Vester-Andersen T, Broby-Johansen U, Bro-Rasmussen F. Perivascular axillary block VI: the distribution of gelatine solution injected into the axillary neurovascular sheath of cadavers. Acta Anaesthesiol Scand. 1986;30:18-22. 